Andrews University

Digital Commons @ Andrews University

Honors Theses

Undergraduate Research

4-5-2019

\title{
Analysis of DL-Amino Acid Ratios in Eggshells Using Reverse Phase-High Pressure Liquid Chromatography
}

Gergana Milkova

Andrews University, gergana@andrews.edu

Follow this and additional works at: https://digitalcommons.andrews.edu/honors

Part of the Biochemistry Commons, and the Organic Chemistry Commons

\section{Recommended Citation}

Milkova, Gergana, "Analysis of DL-Amino Acid Ratios in Eggshells Using Reverse Phase-High Pressure Liquid Chromatography" (2019). Honors Theses. 205.

https://dx.doi.org/10.32597/honors/205

https://digitalcommons.andrews.edu/honors/205

This Project Report is brought to you for free and open access by the Undergraduate Research at Digital Commons @ Andrews University. It has been accepted for inclusion in Honors Theses by an authorized administrator of Digital Commons @ Andrews University. For more information, please contact repository@andrews.edu. 


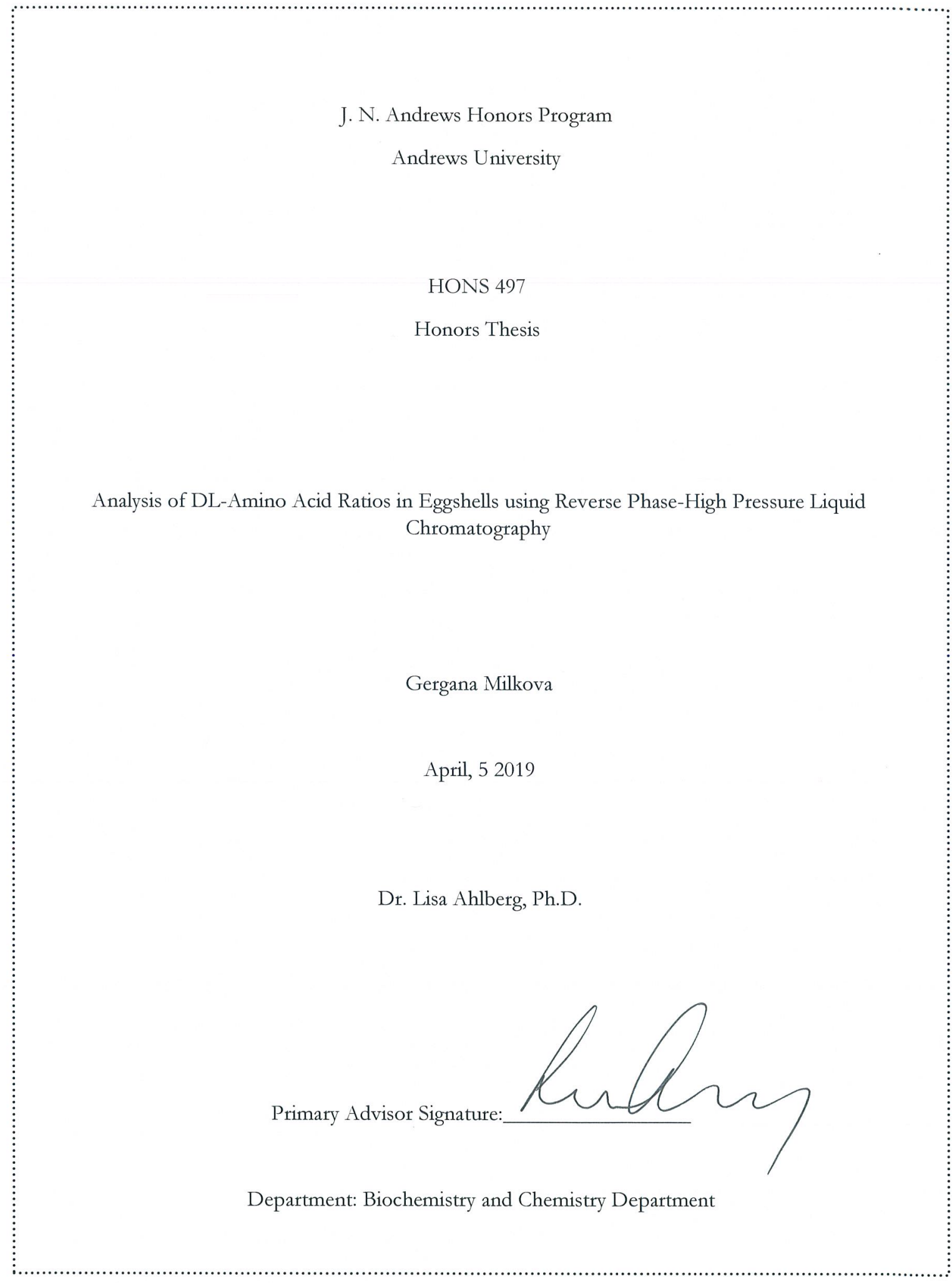


Abstract:

HPLC methodology was developed to determine the concentrations and ratios of $D$ to $L$ amino acids in emu and ostrich eggshells treated with heat at different temperatures. We aimed to determine an internal standard and how ratios were affected under different conditions. An HPLC method was determined that gave consistent retention times and satisfactory enantioseparation. Calibration curves for each amino acid were developed from single and multi-amino acid containing dilution series producing a model that most closely replicates the eggshell-extracted amino acids. 
Introduction:

The ratio of $D$ and $L$ amino acids in eggshells is actually a great story of the conditions to which they were exposed. Emu and ostrich eggshells were treated with heat at different temperatures. While only L-enantiomers of amino acids are present in biologically active systems, during the decomposition of organic material, the spontaneous racemization of amino acids occurs. The rate at which this occurs is influenced by temperature. Because the racemization occurs through a first order reaction, the ratio of $D$ and $L$ enantiomers at different temperatures will vary. Since most proteins in eggshells are found in mamillary sites where calcification occurs, the concentration of amino acids will be preserved. ${ }^{4}$ This will allow for quantification of their concentrations and $d / I$ ratios of the amino acids. A type of chromatography called HPLC was used to quantify these ratios and concentrations.

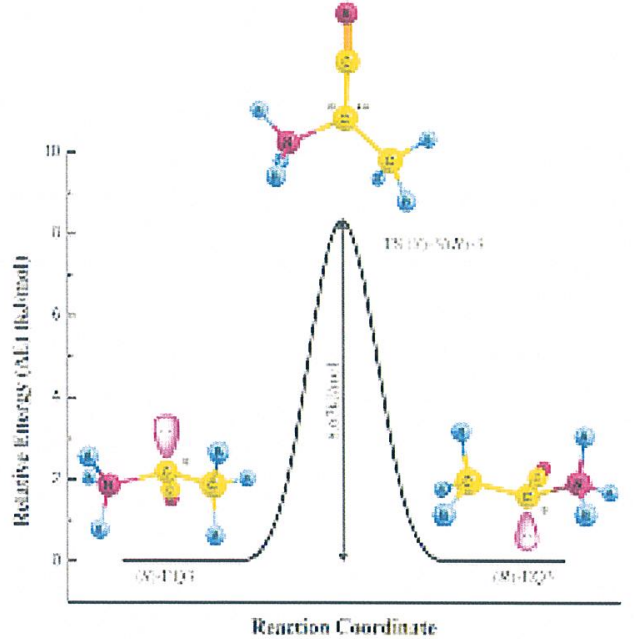

Figure 1: The image below demonstrates the energy diagram for the stereoinversion of a generic amino acids.

When looking at the racemization of amino acids, it occurs through a first order reaction rate. Referencing the Arrhenius equation which relates the rate constant to the activation energy,

$$
k=A e^{-E_{a} / R T}
$$

it can be seen that as the temperature increases, the overall activation energy is approached and the rate constant's magnitude increases. As the rate constant magnitude increases, it means that the reaction would proceed because it crosses the energy barrier.

Furthermore, the mechanism through which racemization occurs, it is an SN1. ${ }^{8}$ The first thing to consider is that the transition state that forms at the activation energy barrier is a carbocation. This can occur under acidic or basic conditions once the $\alpha$-hydrogen is removed. Afterwards, the $\alpha$-hydrogen has an equal probability of attaching to either face of the molecule that will give it $L$ or $D$ spatial configuration. Therefore, when looking at the energy versus reaction coordinate for racemization, it should be symmetrical indicating neither side is energetically favored. ${ }^{8}$ Because each amino acid has a different side-chain, however, when the optimization of geometry occurs at the transition state, the stability of each carbocation could be lower in energy. This would lead to a dip in the plot as shown in Figure.2. This would in turn affect the overall rate constant as well as the amino acid $\mathrm{D} / \mathrm{L}$ ratios. Thus, by developing a methodology using HPLC to first model the environment of the extracted-amino acid from eggshells, an internal standard for temperature in relation to ratios could be developed for the amino acids alongside with actually computing their

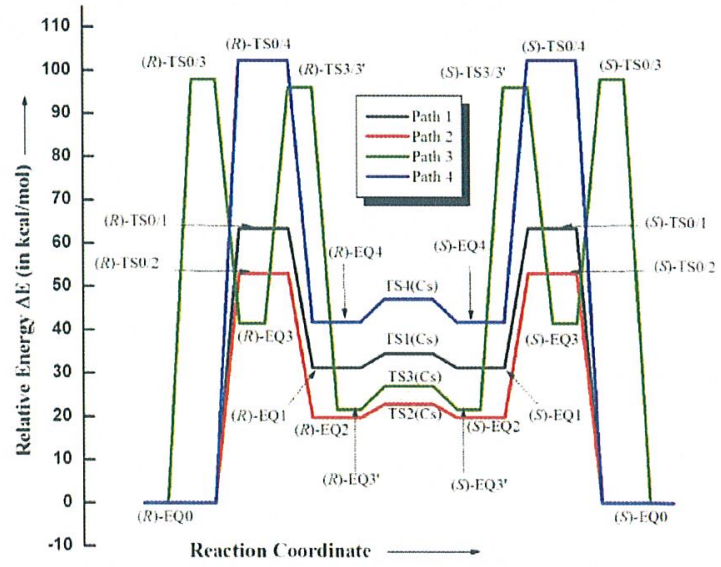

Figure 2: Energy versus reaction coordinate for the four pathways of aspartic acid stereoinversion.? concentrations. This information could then be applied to looking at the systems of eggshells, such as 
nesting sites and determining under what conditions of temperature they existed in. As the eggshells are heated to higher temperatures an overall increase in the D/L-amino acid ratio is predicted to occur. However, with each different amino acid and its functional group, the range of temperatures that will allow for stereoinversion will vary.

The stereoinversion mechanism for aspartic acid was studied where multiple pathways were found as can be seen in Figure.3. Path 1-3 are shown in Figure.3. In path 1, the $\alpha$-carbon was first removed forming a carbocation. ${ }^{5}$ Then an equilibrium structure will form when the proton will either attach to the amine or carbonyl group. As the proton shifts, the transition state will form. Afterwards,

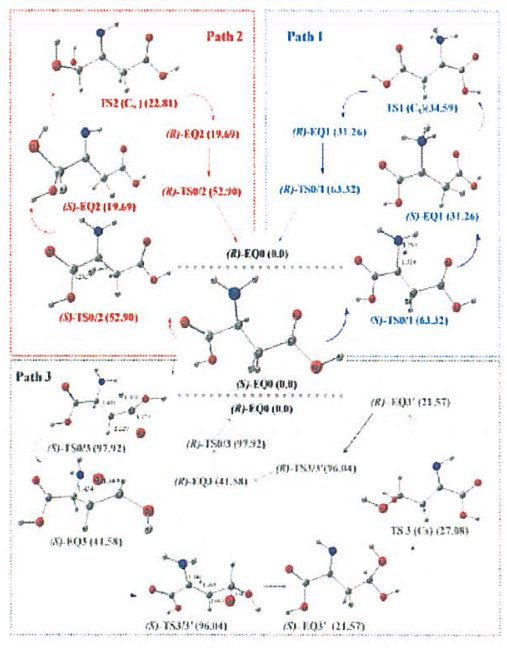

Figure 3: The multiple potential pathways for aspartic acid's stereoinversion are demonstrated above with each of them showing how the $\alpha$-hydrogen would shift around the structure to achieve ${ }^{5}$ stereoinversion. ${ }^{7}$

being formed which is the transition state structure. Figure. 1 shows the energy versus reaction coordinate for a generic amino acid stereoinversion. Because there is only one activation energy to overcome or only one transition state to reach, the likelihood of stereoinversion of alanine occurring, once heated to the right temperature, is much higher than that of molecules such as aspartic acid. This can be also be seen more clearly in Figure. 2 which shows the Energy versus reaction coordinate for the four pathways of aspartic acid stereoinversion. Because of the multiple transition states within each path, there are multiple "activation energies" and transition states. Thus, stereoinversion becomes highly improbable. This is primarily also seen with the carbocation equilibrium followed by another equilibrium structure, stereoinversion will occur from the $L$ to $D$ amino acid. The energy of each state in its mechanisms was determined by geometry optimization in silico. With aspartic acid being a symmetrical structure, it can very easily shift the $\alpha$-proton through its system. The feasibility of this mechanism was tested in conditions of interstellar medium (ISM). Table .1 demonstrates at which temperatures the reaction will have a negative Gibbs Free Energy indicating the probability of reaction progress. However, none of the mechanisms gave a negative Gibbs Free Energy. While none of the mechanisms gave a negative Gibbs Free Energy, it was still determined that "the temperature region of $100-500 \mathrm{~K}$ is more suitable for stereoinversion in aspartic acid to occur". ?

When looking at alanine, there seems to be only one mechanism possible with the $\alpha$-hydrogen removed and carbocation

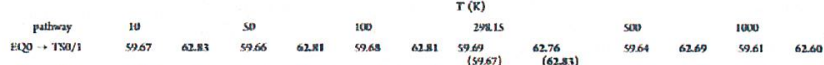

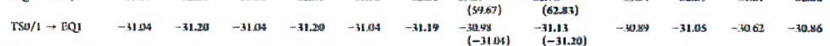

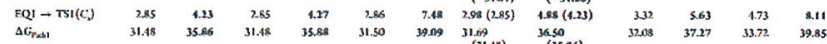

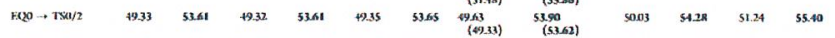

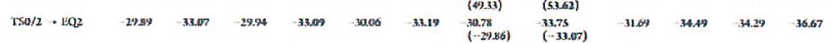

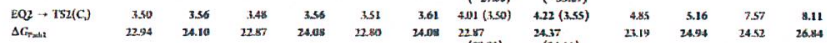

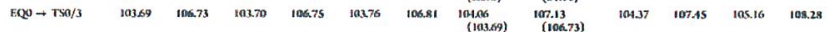

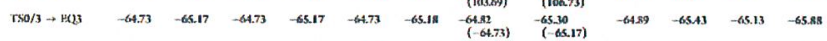

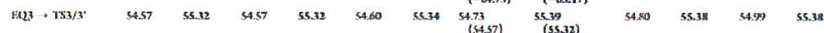

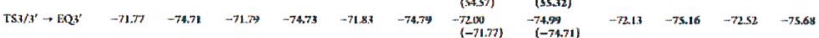

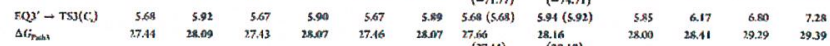

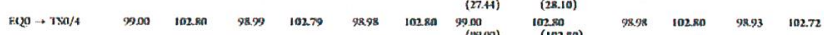

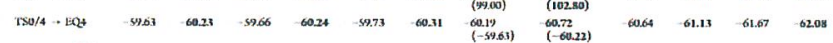

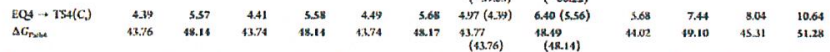

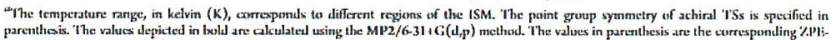


structures formed for each enantiomer being lowest in energy and thus being the most stable structure. This could primarily lead to the pathway stopping somewhere along one of the carbocation structures. More simple structures such as alanine and valine are suspected to only have one pathway for stereoinversion and have an energy schematic as the one in Figure.1. Nonpolar amino acids are predicted to have significantly higher $\mathrm{D} / \mathrm{L}$ ratios than amino acids that follow the pathways in Figure.3.

Methodology: HPLC

The HPLC methodology in this research was first developed by Kaufman and Manley. This methodology is significant because it solved shortcomings of previous HPLC and GC methods. The previously developed HPLC methods achieved separations using "ion exchange chromatography but that could only resolve a single pair of amino acids, isoleucine and alloisoleucine" ${ }^{6}$. GC methods had sample preparations that were time consuming which is a "disadvantage because large inter-sample variability is inherent in some taxa and requires much analysis to reduce the uncertainty involved in deriving robust D/L radios", ${ }^{4}$ The HPLC instrument used is the Agilent 1260 Infinity II LC System. The column used is Agilent Poroshell 120 EC-C18 $2.7 \mu \mathrm{m} 4.6 \times 150 \mathrm{~mm}$. The HPLC standard for this specific column is containing 0.028 grams Uracil, 0.1020 grams Phenol, 0.0122 grams 4-Chloro Nitrobenzene and 0.0202 grams Naphthalene in a mobile phase that is $70 \%$ acetonitrile and $30 \%$ water solution. The column standard was run to ensure the column was functioning properly and is run regularly to ensure the column quality is not degrading. The standard method for the column with the flow rate and mobile phase is listed in the table below.

\begin{tabular}{|c|c|c|c|c|}
\hline $\begin{array}{c}\text { Time } \\
(\mathrm{min})\end{array}$ & $\begin{array}{c}\text { Water (Percent } \\
\text { composition) }\end{array}$ & $\begin{array}{c}\text { Acetonitrile (Percent } \\
\text { composition) }\end{array}$ & $\begin{array}{c}\text { Flow rate } \\
(\mathrm{ml} / \mathrm{min})\end{array}$ & $\begin{array}{c}\text { Max } \\
\text { Pressure }\end{array}$ \\
\hline 0 & 30 & 70 & 1.8 & 400 \\
\hline 3 & 30 & 70 & 1.8 & 400 \\
\hline
\end{tabular}

The column is then washed with the method below.

\begin{tabular}{|c|c|c|c|c|}
\hline $\begin{array}{c}\text { Time } \\
(\mathrm{min})\end{array}$ & $\begin{array}{c}\text { Water (Percent } \\
\text { composition) }\end{array}$ & $\begin{array}{c}\text { Methanol (Percent } \\
\text { composition) }\end{array}$ & $\begin{array}{c}\text { Flow rate } \\
(\mathrm{ml} / \mathrm{min})\end{array}$ & $\begin{array}{c}\text { Max } \\
\text { Pressure }\end{array}$ \\
\hline 0 & 0 & 100 & 0.8 & 400 \\
\hline 10 & 0 & 100 & 0.8 & 400 \\
\hline 11 & 50 & 50 & 0.8 & 400 \\
\hline 21 & 100 & 0 & 0.8 & 400 \\
\hline
\end{tabular}

The HPLC UV-Vis detector was used to be able to visualize and determine the location of the amino acids with relation to time. The greatest absorption from the UV-Vis spectra of the amino acids came out at $338 \mathrm{~nm}$ and $280 \mathrm{~nm}$. The settings used for the UV-Vis detector on the HPLC are summarized in the table below.

\begin{tabular}{|c|c|c|c|}
\hline Wavelength $(\mathrm{nm})$ & Band Width $(\mathrm{nm})$ & Reference Wavelength $(\mathrm{nm})$ & Reference Bandwidth $(\mathrm{nm})$ \\
\hline 338 & 10 & 390 & 20 \\
\hline 338 & 10 & 390 & 20 \\
\hline
\end{tabular}


Precolumn derivation was performed using o-phthaldialdehyde (OPA) and $\mathrm{N}$-isobutyryl-L-cysteine (IBLC). The concentration of OPA is $0.17 \mathrm{M}$ and the concentration of IBLC is $0.26 \mathrm{M}$. Figure. 1 shows the reaction between OPA, a generic amino acid and IBLC. It is the OPA group that allows for UV-Vis detection because of its high conjugation and IBLC that allows for the separation and differentiation of the two enantiomers of the amino acid. ${ }^{5}$ The OPA and IBLC solutions were both made in $1 \mathrm{M}$ potassium borate buffer with a $\mathrm{pH}$ of 10.4 .

The amino acid solutions have concentrations of $0.001 \mathrm{M}$ and are dissolved in $0.05 \mathrm{M}$ $\mathrm{HCl}$. The two mobile phases used during the amino acid runs is acetate buffer and methanol. The acetate

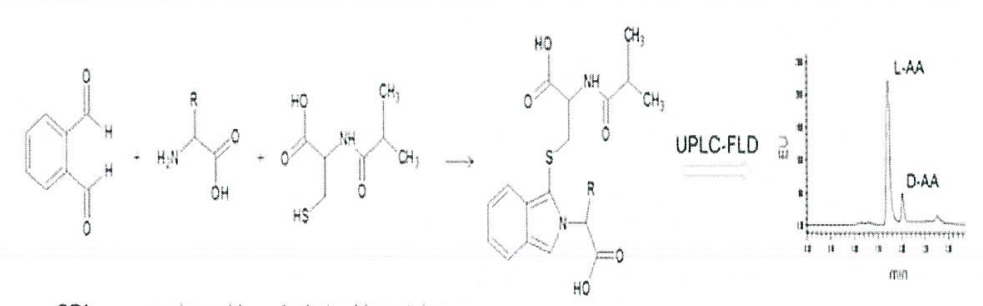

OPA amino acid isobutyry|L.L.cysteine

Figure 2: In this reaction, the derivatization between $O P A$, an amino acid and IBLC is shown. It is the thiol group that is responsible for the enantioseparation of DL-amino acids. The addition of the OPA to the complex allows for UV detection. ${ }^{10}$

buffer had a $\mathrm{pH}$ of 4 . The method is listed in the table below.

\begin{tabular}{|c|c|c|c|c|}
\hline $\begin{array}{c}\text { Time } \\
(\mathrm{min})\end{array}$ & $\begin{array}{c}\text { Acetate Buffer (Percent } \\
\text { composition) }\end{array}$ & $\begin{array}{c}\text { Methanol (Percent } \\
\text { composition) }\end{array}$ & $\begin{array}{c}\text { Flow rate } \\
(\mathrm{ml} / \mathrm{min})\end{array}$ & $\begin{array}{c}\text { Max } \\
\text { Pressure }\end{array}$ \\
\hline 0 & 76.6 & 23.3 & 1.0 & 400 \\
\hline 30 & 23.3 & 76.7 & 1.0 & 400 \\
\hline
\end{tabular}

The injection method developed is listed in the table below.

\begin{tabular}{|c|c|}
\hline Function & Parameter \\
\hline Draw & $\begin{array}{c}\text { Draw } 2.5 \mu \text { from location vial "'” (pH } 10.4 \text { borate buffer) with default speed and default } \\
\text { offset }\end{array}$ \\
\hline Draw & Draw $1 \mu$ from sample with default speed and default offset \\
\hline Mix & Mix $3.5 \mu \mathrm{l}$ from air with default speed for 5 times \\
\hline Wait & Wait 0.2 minutes \\
\hline Draw & Draw $0.5 \mu$ from location Vial "" OPA solution with default speed and default offset \\
\hline Mix & Mix $4 \mu$ from air with default speed for 10 times \\
\hline Draw & Draw $0.5 \mu$ l from location Vial "' IBLC solution with default speed and default offset \\
\hline Mix & Mix $4.5 \mu \mathrm{l}$ from air with default speed for 10 times \\
\hline Inject & Inject \\
\hline Wait & Wait 0.1 minutes \\
\hline Valve & Switch valve to "Bypass" \\
\hline
\end{tabular}


The injector program was designed so that adequate time was provided for the mixing between the potassium borate buffer, OPA/IBLC derivatizing agents and the sample. The final step in the injector program was included to bypass the injector loop. This will prevent extra column volume to enter causing dispersion of the sample and broadening of the peaks. When looking at Figure.5, it shows how the injector loop's length can be shortened or prevent excess volume to enter the column. In order to prevent degradation and not allow for precipitation to occur on the column, in between or after usage of the column the ${ }^{1}$ following method was run on the column.

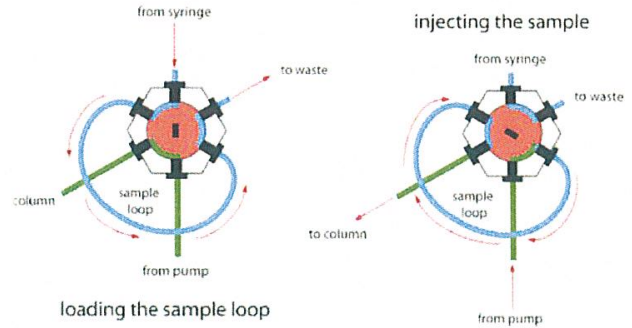

Figure 3: Diagram of how injector program's loop can be changed or shortened as sample/derivatizing reaction is injected ${ }^{1}$

\begin{tabular}{|c|c|c|c|c|}
\hline $\begin{array}{c}\text { Time } \\
(\mathrm{min})\end{array}$ & $\begin{array}{c}\text { Water (Percent } \\
\text { composition) }\end{array}$ & $\begin{array}{c}\text { Methanol (Percent } \\
\text { composition) }\end{array}$ & $\begin{array}{c}\text { Flow rate } \\
(\mathrm{ml} / \mathrm{min})\end{array}$ & $\begin{array}{c}\text { Max } \\
\text { Pressure }\end{array}$ \\
\hline 0 & 0 & 100 & 0.8 & 400 \\
\hline 10 & 0 & 100 & 0.8 & 400 \\
\hline 11 & 50 & 50 & 0.8 & 400 \\
\hline 21 & 100 & 0 & 0.8 & 400 \\
\hline
\end{tabular}

Methodology: Lab Procedure: Diagnostics of pressure with different mobile phases on column

The first few issues to be encountered using the HPLC was the predominantly high pressure. This caused for the HPLC system's software to crash any possible run. Running the amino acid mobile phase caused for the pressure to reach as high as 420 bar with the HPLC system having a threshold of 400 bar. The pressure was slightly better off with column's standard mobile which allowed for the HPLC to be run with a flow rate $0.8 \mathrm{ml} / \mathrm{min}$ and a pressure reaching an upper high of 397 bar. However, the column was no where near optimal performance with the recommended flow rate with that mobile phase being 1.5 $\mathrm{ml} / \mathrm{min}$. When running the system to waste, the pressure was still too high with an upper limit of 70 bar.

\section{Methodology: Lab Procedure: Instrument Maintenance}

When first beginning to work with the instrument, the pressure of the solvents was far too high to actually run the necessary mobile phase to receive the desired chromatogram. The first step was to first change the filters of the HPLC system. The first filter to be changed was the in-line filter. When looking at the diagram in Figure.6, the in-line filter that was replaced was the one that blocks off any extra particulates that come from the sample (Structure \#1). When running the $70 \% \mathrm{ACN} / 30 \%$ distilled water mobile phase through the waste, there wasn't a decrease in pressure to be observed. This is

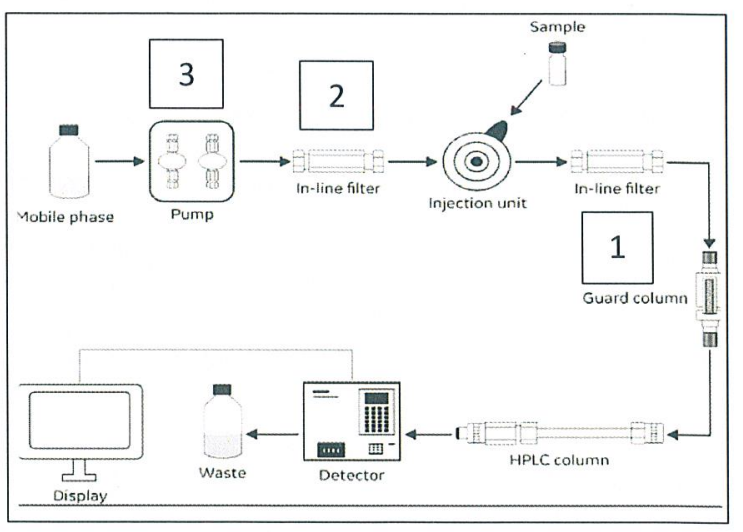

Figure 4: The HPLC diagram showing where each of the filters were located within the other structures. ${ }^{3}$ 
because the system wasn't circulated through the column or the injector program.

The second filter that was changed was the high -pressure filter. The high- pressure filter is found at the secondary pipette where the capillary connection was first removed. The filter assembly was then loosened with a wrench. The high-pressure filter assembly was then freely taken out (schematic shown in Figure.7) where the gold ceiling cap was taken off the configuration and the PTFE frit was changed (Structure \#2 in Figure.6). The PTFE's function

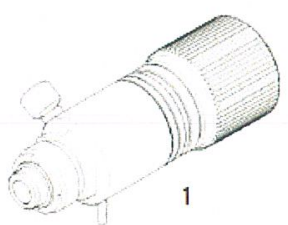

2 3

Figure 5: The PTFE'S structure is shown in this diagram where the location of the filter, labeled structure 2 is shown.10 is "to retain ion exchange within microcolumns"10 which allows for organic solvent filtration and is "chemically inert, nonleaching, and non-reactive for high sensitivity analysis" ${ }^{10}$. This frit is an example of a precolumn filter which are important in preserving the integrity of the HPLC columns against particulate matter build-up. What the changing of this particular frit does is in contributing is catching any ions present. This is very important because in this experiment organic solvents such as the mobile phase, methanol is mixed with acetate buffer. Furthermore, if excessive ions from the mobile phase are not caught, they will build up on the column or through the HPLC system and cause back-pressure. When running the mobile phase composition of $70 \%$ acetonitrile and $30 \%$ distilled water at $1.8 \mathrm{ml} / \mathrm{min}$ through the column, the pressure was far above 400 bar. After the change of the filter, the pressure dropped to just slightly below 400 bar indicating that the changing of the filter had some significance. When running the mobile phase through waste, the pressure for 1 $\mathrm{ml} / \mathrm{min}$ dropped to approximately 4 bar where before it ranged from 45-70 bar. However, when running acetate buffer/methanol, even at $0.8 \mathrm{ml} / \mathrm{min}$ going to waste the pressure would be approximately 70 bar.

The pump seals (Structure \#3 in Figure.6) were also replaced as well as their inside housing, support rings and seal holders being cleaned with isopropyl alcohol as to remove any particulates that might have or will clog up the seals. Changing the pump seals is important because the HPLC system is constantly being flushed with aqueous salt buffers that can clog them up alongside with other particulate matter that is unavoidably present. This build-up will eventually be carried through the lining onto the column and increase the back pressure. Once the pump seals were switched out, the pressure using amino acid run's mobile phase from 397-420 bar to 320-380 bar. When equilibration time and preparatory run is included the pressure has been observed to drop down to as low as 250-300 bar as well as remaining relatively constant throughout the run. After running the amino acid mobile phase through waste, the pressure was as low as 4 bar. As mentioned previously when changing the mobile phase to the amino acid one, there was the increase in pressure when it went to waste. This could be explained by particulate matter simply building up again over time. But also, when looking at Figure.6, the system maintenance was performed backwards. The in-line filter for the sample was changed first followed by the in-line filter for the pump and finally the pump seals filter being changed out. Since potentially all these three areas not being changed out added to pressure and since we are working backwards, until the pump seals were switched out the back pressure would not have dropped. 
Results and Discussion:

The first amino acid solution to be run was DL-tyrosine which was composed of 0.0016 grams and dilute to $10 \mathrm{~mL}$ using $0.05 \mathrm{M} \mathrm{HCl}$. The mobile phase that was run was

\begin{tabular}{|c|c|c|c|c|}
\hline $\begin{array}{c}\text { Time } \\
(\mathrm{min})\end{array}$ & $\begin{array}{c}\text { Water (Percent } \\
\text { composition) }\end{array}$ & $\begin{array}{c}\text { Acetonitrile (Percent } \\
\text { composition) }\end{array}$ & $\begin{array}{c}\text { Flow rate } \\
\text { (ml/min) }\end{array}$ & $\begin{array}{c}\text { Max } \\
\text { Pressure }\end{array}$ \\
\hline 0 & 30 & 70 & 1.8 & 400 \\
\hline 3 & 30 & 70 & 1.8 & 400 \\
\hline
\end{tabular}

The issues that arose with running this mobile phase was that as the mobile phase gradient was occurring, with the transition between acetonitrile to methanol, the increase in viscosity caused for a major deviation in the baseline. Because the intensity of the chromatogram's peaks is determined by integrating over the height of the peak, with a deviation from the baseline, an inaccurate intensity will be measured. Since the future calibration curves will function off of the principle that the intensity is proportional to the concentration, with an inaccurate intensity, an inaccurate concentration will be obtained. Also, the baseline deviations are not always constant. The pressure issues thus challenged the robustness of the method. Because the column equilibration is very slow with the changing composition of the mobile phase, a preparatory run was introduced into the methodology. Before allowing for a run to proceed, adequate time was provided for the pressure to equilibrate with just the initial mobile phase of $76.7 \%$ acetate buffer and $23.3 \%$ methanol. Then a run was introduced in which the mobile phase was run backwards and forwards as shown below.

\begin{tabular}{|c|c|c|c|c|}
\hline $\begin{array}{c}\text { Time } \\
(\mathrm{min})\end{array}$ & $\begin{array}{c}\text { Acetate Buffer (Percent } \\
\text { composition) }\end{array}$ & $\begin{array}{c}\text { Methanol (Percent } \\
\text { composition) }\end{array}$ & $\begin{array}{c}\text { Flow rate } \\
\text { (ml/min) }\end{array}$ & $\begin{array}{c}\text { Max } \\
\text { Pressure }\end{array}$ \\
\hline 0 & 76.7 & 23.3 & 1.0 & 400 \\
\hline 30 & 23.3 & 76.7 & 1.0 & 410 \\
\hline 52 & 76.7 & 23.3 & 1.0 & 410 \\
\hline
\end{tabular}

The baseline deviation significantly improved as can be seen in Figure.6. ${ }^{1}$

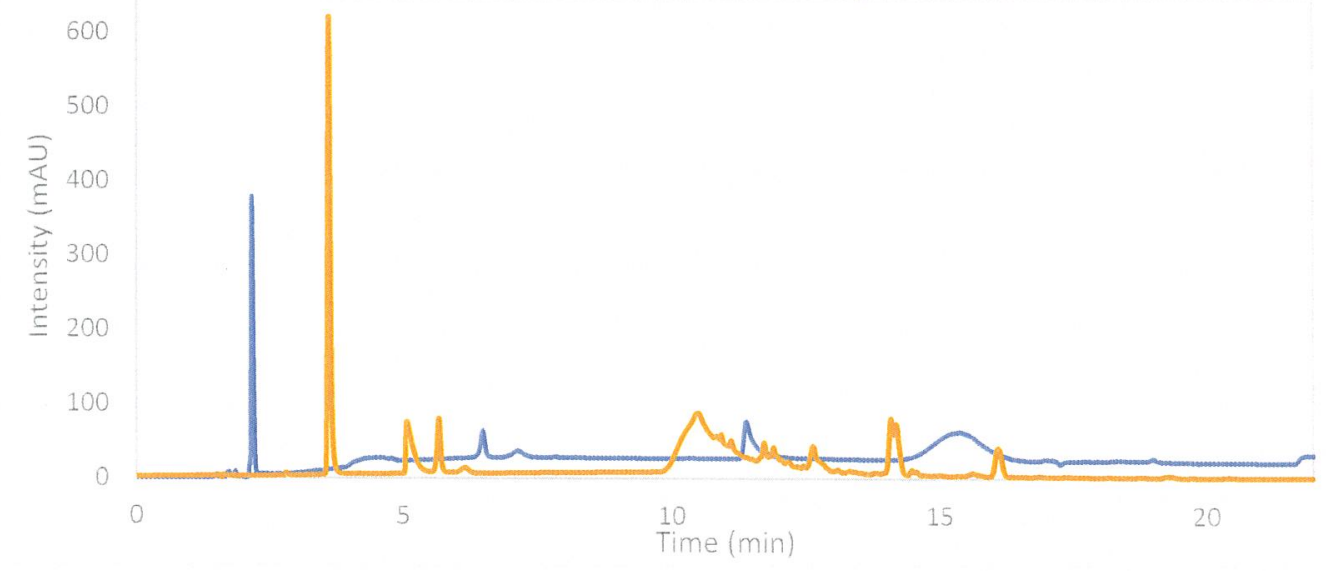

Figure 6: The intensity vs time chromatograms of the tyrosine before and after the implementation of the preparatory column prep. 
When beginning to run multiple amino acid solutions, however the same amino acid run began to appear. This meant that something as pegging out the detector in that, the detector might not actually be seeing the amino acid run but rather something else that is either precipitated onto the column or somewhere within the HPLC system. A flow chart was created to be help during the troubleshooting in pin-pointing the problem.

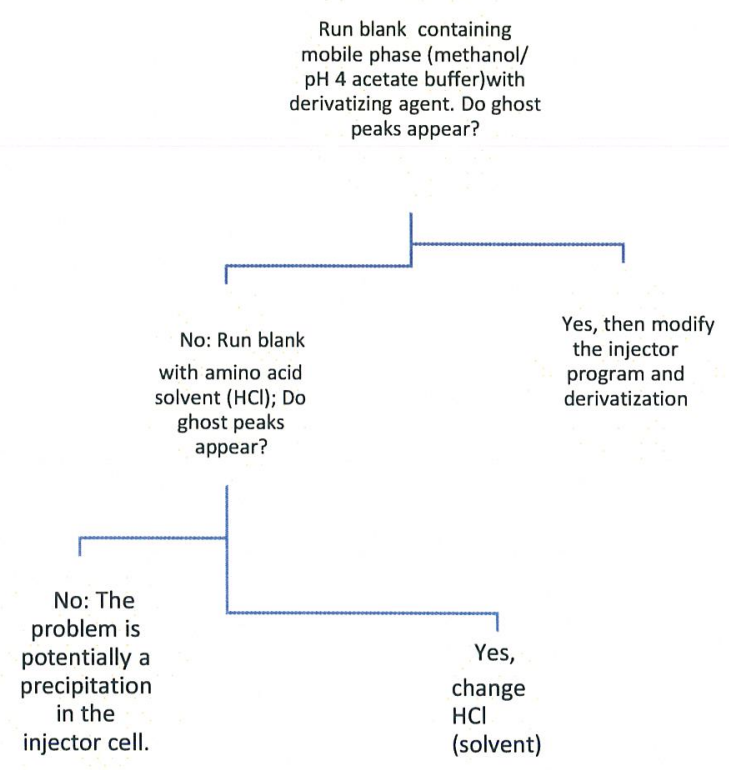

When running only the mobile phase with no injection method, the run was completely blank except for two ghost peaks

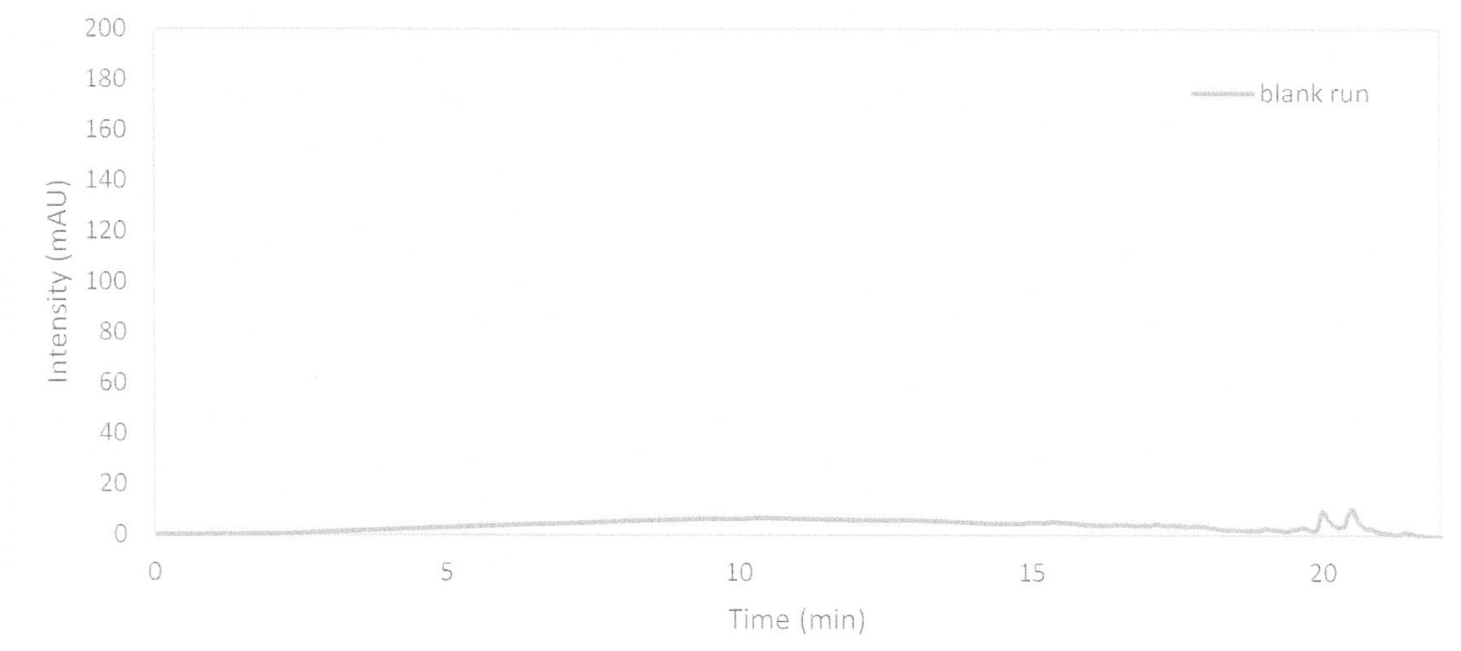

Figure 7: The chromatogram that was collected when only the mobile phase without the injector method was run 
Afterwards, the injector program was run with blank which simply contained methanol. The run that was obtained

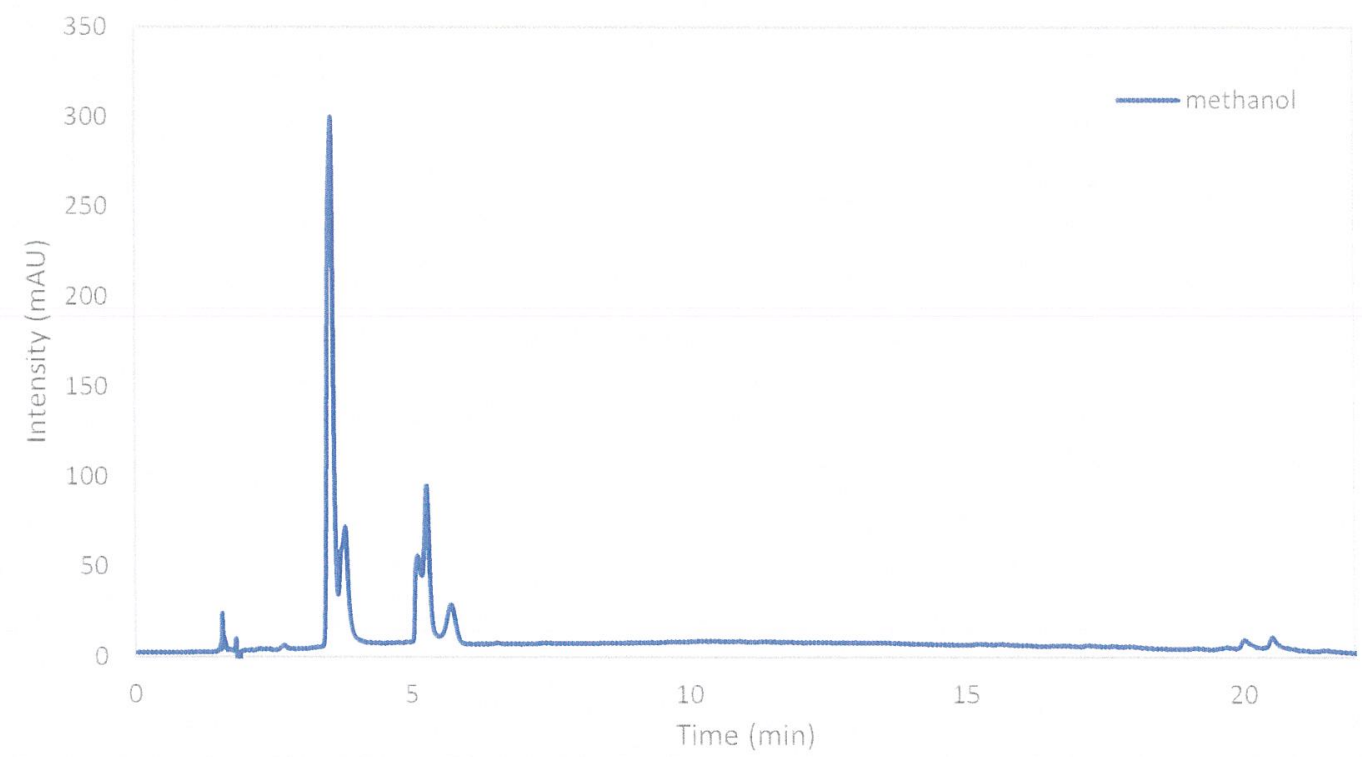

Figure 8: The chromatogram that was processed when a blank, methanol was run with the injection methods and mobile phase

This helped conclude that nothing had actually precipitated onto the column but rather the issue was with the injector, injector program or even the flow cell. When superimposing these two runs with the actual amino acid run, the plot that is received is Figure.11

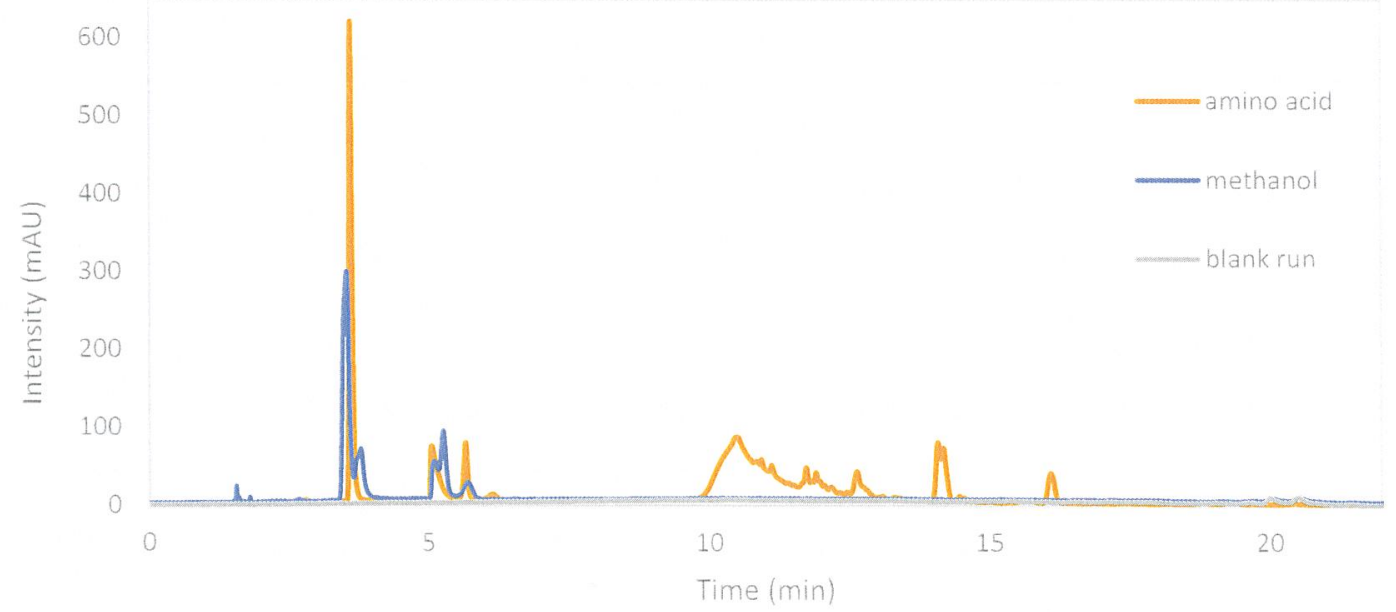

Figure 9: All of the plots from troubleshooting were superimposed so as to better isolate the ghost peaks, which are seen in between the 10-15 minutes time frame

According to this plot, the major peak's origin that appears at the retention time of approximately 3.648 minutes, however later on they are ghost peaks that appear as well as tailing that can be seen in the widest peaks that starts at approximately 10 minutes. The only difference or solvent that was present 
with the amino acid run that was not before is the hydrochloric acid. The hydrochloric acid used to dissolve the amino acids and seems to be the most plausible or possible origin of those ghost peaks. When switching out the columns with a different brand new one, the same peak that appears at 3.648 minutes is immediately picked up by the detector. The precolumn or column protector was determined to have something precipitated onto to it and was switched out. Solving the challenges listed above should allow for us to be able to obtain single amino acid chromatograms. Figure.12 below demonstrates single amino acid runs superimposed onto each other.

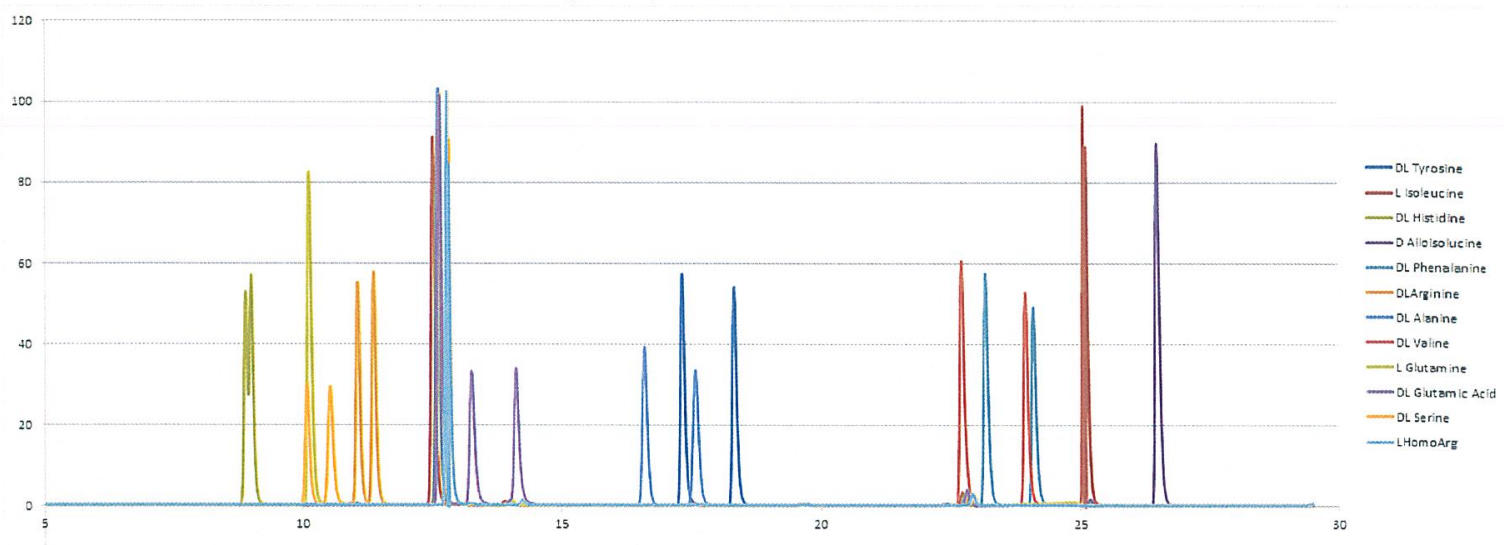

Figure 10: Single amino acid runs superimposed onto each other

Eventually once multi-amino acid runs are run, a chromatogram should be collected in which all of them have good resolution and enantioseparation. As can be seen in the plot above, many of the amino acids are already overlapping. The method for the amino acid run will need to be modified so that each amino acid is resolved. This methodology will ensure that the experimental data is a model that most closely resembles the amino acids that will be extracted from the eggshell matrices.

Conclusion:

The potential problem as to why the same chromatogram was appearing for each amino acid run could stem from either injector needle precipitation, contaminant in the flow cell or the something having precipitated in the pre-column. The next few steps to take would be to switch out the pre-column. Also running every single solution through UV-Vis to see exactly what is being absorbed. Furthermore, studying one of the simpler amino acids such as alanine and valine's racemization mechanism and determining a temperature range for which $\mathrm{D} / \mathrm{L}$ ratio will increase using geometry optimization, will allow for a better hypothesis to be developed. 


\section{References}

1. Harvey, D., Loop Injector for HPLC.

https://community.asdlib.org/imageandvideoexchangeforum/2013/08/02/loop-injector-for-hplc

2. How to identify, isolate, and correct the most common HPLC problems.

https://www.sigmaaldrich.com/technical-documents/articles/analytical/hplc-troubleshooting-

guide.html\#18 (accessed November 5).

3. HPLC Pain Points https://www.gelifesciences.com/en/us/solutions/lab-filtration/knowledgecenter/hplc-pain-points-part-1.

4. Bada, J. L.; Helfman, P. M., Amino acid racemization dating of fossil bones. World archaeology 1975, 7 (2), 160-73.

5. Brueckner, H.; Westhauser, T.; Godel, H., Liquid chromatographic determination of D- and Lamino acids by derivatization with o-phthaldialdehyde and N-isobutyryl-L-cysteine. Applications with reference to the analysis of peptidic antibiotics, toxins, drugs and pharmaceutically used amino acids. 1995; Vol. 711, p 201-15.

6. Kaufman, D.; F. Manley, W., A new procedure for determining $D L$ amino acid ratios in fossils using Reverse Phase Liquid Chromatography. 1998; Vol. 17, p 987-1000.

7. Kaur, R.; Rani, N.; Vikas, Gas-Phase Stereoinversion in Aspartic Acid: Reaction Pathways, Computational Spectroscopic Analysis, and Its Astrophysical Relevance. 2018; Vol. 3, p 14431-14447. 8. Kaur, R.; Vikas, Mechanisms for the inversion of chirality: global reaction route mapping of stereochemical pathways in a probable chiral extraterrestrial molecule, 2-aminopropionitrile. The Journal of chemical physics 2015, 142 (7), 074307.

9. Müller, C.; R Fonseca, J.; Bader, T.; Krauss-Etschmann, S.; Schmitt-Kopplin, P., Enantioseparation and selective detection of $D$-amino acids by ultra-high-performance liquid chromatography/mass spectrometry in analysis of complex biological samples. 2013; Vol. 1324.

10. Technologies, A., Agilent 1260 Infinity Isocratic Pump and Quaternary Pump. Agilent Technologies. https://www.agilent.com/cs/library/usermanuals/Public/G1310-90016 Iso-QuatPumpB USR EN.pdf. 